\title{
James C. Carr, Timothy J. Carroll: Magnetic resonance angiography: principles and applications
}

\author{
Springer, New York, Dordrecht, Heidelberg, London, 2012, ISBN: 978-1-4419-1685-3
}

\author{
Daniel Badger
}

Published online: 23 May 2013

(C) Australasian College of Physical Scientists and Engineers in Medicine 2013

This book consists of two sections, each containing a number of independent chapters by different authors. The first section covers MRI and magnetic resonance angiography (MRA) techniques and the second section focuses more on application of MRA to imaging specific parts of the body.

Part one starts with an excellent treatment of the basic principles of MRI and of MRA. The introduction to the magnetic resonance effect and image formation is very clear and well illustrated and is possibly the best such introduction I have read. It covers the Magnetic resonance effect and the T1, T2 and proton density contrast mechanisms in detail and then goes on to explain image formation, SNR and parallel imaging. There is a slight issue in that pulse sequences-especially those in common use such as fast gradient echo, are mentioned but not explained, even though mentions of them are then scattered extensively through the rest of the book.

The MRA section of the first chapter is also clear, covering contrast MRA, time of flight (TOF) MRA and Phasecontrast MRA, but it does jump dramatically in assumed knowledge of MRI principles, for example the term "spoiled" is used assuming the reader knows what it means without the term having being explained, or even mentioned in the previous section. However, contrast material, TOF and phase contrast are well explained and should be understandable to any reader with a good prior knowledge of MRI imaging. It is a little puzzling that the chapter should contain such a good introductory section then jump to assuming the reader already has a good grasp of MRI. One

D. Badger $(\bowtie)$

Department of Nuclear Medicine, The Queen Elizabeth Hospital, Adelaide, SA, Australia

e-mail: daniel.badger@health.sa.gov.au could reasonably assume that most readers of this type of book would already have a high level of understanding, in which case why have such a basic introduction at all?

The subsequent chapters of section one cover different MRA techniques in detail, with chapters on TOF, Phasecontrast and Contrast-enhanced MRA. There are also chapters on many other technical issues, including lowdose contrast angiography, vessel wall imaging, cardiac imaging, high strength magnets, parallel imaging, different contrast agents and motion compensation. These chapters are generally well written and clear. As they are written as separate chapters rather than as a coherent whole, many parts of the chapters are devoted to introductory segments which repeat or rehash previously covered work. For example, chapter six: "Flow Dependent Nonconstrast MR Angiography", has introductory sections on TOF and Phase-contrast MRA which are well covered by the basic principles chapter (chapter 1), as well as the chapters on TOF (chapter 2) and Phase-contrast MRA (chapter 3). This sort of thing is repeated throughout the book

Part two of the book concentrates on clinical applications of MRA, with discussions on the use of various MRA techniques for visualisation of different pathologies and imaging of blood vessels in different areas of the body including the heart, lungs, kidneys, abdomen and extremities. This part consists again of separate chapters with no linking between them and while this means material is repeated, it is possibly of more practical use to have a reference in which each section is self contained. After all, it seems unlikely that anyone not doing a book review would read this work from cover to cover, but would instead go to straight to the chapter covering the area of interest at the time.

Overall, the book seems to thoroughly cover the field of MRA and would be a useful reference to any person who is interested or working in MRA. 